\title{
Etiology and antibiotic susceptibility patterns of bacteria collected from urinary tract infections in the ASL3 in Genoa
}

\author{
Manuela Fedele, Paolo Piazzai, Orietta Illiberi, Alida Galleano, Susanna Oddera, Elisabetta Schellino, \\ Stefania Urbini, Luigi Carlo Bottaro \\ ASL3 Ospedale San Carlo, Genova
}

Key words: Antimicrobial resistance, urinary pathogens, epidemiology

Eziologia delle infezioni batteriche urinarie ed antibiotico-resistenza nell'ASL3 Genovese

\section{SUMMARY}

An epidemiological study addressed to identify the pathogens collected from urine samples and their antibiotic susceptibility patterns was conducted. From January 2008 to May 2009 56,435 urine samples were processed in the Clinical Microbiological Laboratory of the ASL3 in Genoa.

Materials and methods. Urine samples were firstly screened by automated equipment Uroquik (ALIFAX). All urine cultures with microbial $\geq 10^{5} \mathrm{CFU} / \mathrm{ml}$ were seeded on Chromagar Orientation (BD) and incubated at $37^{\circ} \mathrm{C}^{\circ}$. The identification of the isolates and the evaluation of their susceptibility to antibiotics were determined by the automated system Vitek 2 (bioMérieux).

Results. About 33\% (18,543) of the urine samples gave positive results. The number and frequency of the microorganisms collected was: I3,379 (72\%) Gram-negative including 9179 (69\%) E.coli, I382 (I0\%) Klebsiella spp, I209 (9\%) Proteus spp, 445 (3\%) Pseudomonas spp, and other species II 64 (9\%), 4942 (27\%) Gram positive which included 3615 (73\%) Enterococcus spp, 82I (I7\%) Staphylococcus spp, 506 (I0\% ) Streptococcus spp and 222 (I\%) fungi. In E. coli the incidence of susceptible strains ranged between $90-96 \%$ for gentamicin, fosfomycin, nitrofurantoin, piperacillin-tazobactam, between $\mathbf{8 7 - 8 9 \%}$ for the cefepime, cefotaxime, ceftazidime, about $70 \%$ for quinolones ciprofloxacin, norfloxacin, and trimethoprim-sulfamethoxazole, $62 \%$ for piperacillin, about $50 \%$ to ampicillin. For Klebsiella spp and Proteus spp the percentage of susceptible strains ranged from $95-99 \%$ to piperacillin-tazobactam, gentamicin, and respectively $93 \%$ and between $68-52 \%$ for third-generation cephalosporins cefotaxime and ceftazidime and fluoroquinolones ciprofloxacin and norfloxacin.

Conclusions. Present findings indicate that among the Enterobacteriaceae, E. coli, cause most of UTI and in vitro resulted susceptible various antibiotics. There was an increased resistance to fluoroquinolones among community-acquired $E$. coli and Proteus spp. A periodical epidemiological study will be necessary to monitor the evolution toward resistance to antibiotics of the strains collected from urinary tract infections.

\section{INTRODUZIONE}

Questo studio, condotto da gennaio 2008 a maggio 2009 nell'ambito del Dipartimento di Patologia Clinica ASL3 Genovese, ha valutato sia la frequenza degli isolati batterici responsabili delle I.V.U. che la loro sensibilità e resistenza nei confronti delle diverse classi di antibiotici.

\section{METODI}

Sono stati analizzati 56.435 campioni di urina prelevati da pazienti ricoverati e ambulatoriali esterni. I campioni sono stati monitorati per due ore con apparecchio automatizzato Uroquik (ALIFAX). Tutte le urinocolture con carica microbica $\geq 10^{5}$ U.F.C./ml sono state seminate su Chromagar Orientation (BD) ed incubate a $37^{\circ} \mathrm{C}$. L'identificazione dei ceppi isolati e la sensibilità agli antibiotici sono state determinate con il sistema automatizzato Vitek 2 (bioMérieux).

\section{RISULTATI}

Dall'analisi dei dati 18.543 campioni di urina (33\%) sono risultati positivi. La frequenza dei microrganismi isolati è stata di 72\% (13.379) Gram-negativi che includevano 9.179 (69\%) E.coli, 1.382 (10\%) Klebsiella spp, 1.209 (9\%) Proteus spp, 445 (3\%) Pseudomonas spp, 1.164 (9\%) altri 4.942 (18\%) e Gram-positivi di cui 3.615 (73\%) Enterococcus spp, 818 (17\%) Staphylococcus spp, 504 (10\%) Streptococcus spp e 222 (1\%) miceti.

I risultati della sensibilità agli antibiotici sono riportati nelle Tabelle 1 e 2

Per E.coli la sensibilità è risultata compresa tra il 90-96\% per gentamicina, fosfomicina, nitrofurantoina, piperacillina-tazobactam, compresa tra l'87-89\% per cefepime, cefotaxime, ceftazidime, di circa il 70\% per i chinolonici ciprofloxacina, norfloxacina, e per trimetoprim-sulfametossazolo, del $62 \%$

Tabella I. Distribuzione delle percentuali di resistenza agli antibiotici tra i principali patogeni urinari Gram-negativi isolati in questo studio.

\begin{tabular}{|c|c|c|c|c|}
\hline Antibiotico & E. coli & Klebsiella spp & Proteus spp & Pseudomonas spp \\
\hline Ampicillina & 53 & 99 & 70 & $\mathrm{nv}$ \\
\hline Amox+Ac. Clav. & 26 & II & 37 & nv \\
\hline Amikacina & 0 & 15 & I & 13 \\
\hline Gentamicina & 10 & 4 & 28 & 32 \\
\hline Cefotaxime & II & 7 & 37 & $\mathrm{nv}$ \\
\hline Ceftazidime & $\mathrm{II}$ & 7 & 32 & 28 \\
\hline Cefaclor & 54 & 13 & 51 & nv \\
\hline Cefalotina & 47 & 20 & 55 & $\mathrm{nv}$ \\
\hline Cefepime & 13 & 6 & 22 & 21 \\
\hline Ciprofloxacina & 30 & 6 & 48 & 47 \\
\hline Norfloxacina & 29 & 7 & 39 & 43 \\
\hline Fosfomicina & 9 & 36 & 48 & nv \\
\hline Piperacillina & 38 & 80 & 48 & 18 \\
\hline Piper+tazob & 4 & 5 & 4 & 9 \\
\hline Trimet+sulfam & 29 & 12 & 60 & nv \\
\hline Nitrofurantoina & 6 & 60 & 100 & $\mathrm{nv}$ \\
\hline
\end{tabular}

Nv, non valutabile a causa della resistenza intrinseca

\section{Corresponding author: Paolo Piazzai}

ASL3 Ospedale San Carlo

16158 Genova-Voltri - P.le Giannasso 2

E-mail: paolo.piazzai@asl3.liguria.it 
Tabella 2. Distribuzione delle percentuali di resistenza agli antibiotici tra i principali patogeni urinari Gram-positivi isolati in questo studio.

\begin{tabular}{lccc}
\hline Antibiotico & Enterococcus spp & Staphylococcus spp & Streptococcus spp \\
\hline Ampicillina & 23 & NC & I \\
\hline Amox+Ac. Clav. & 3 & 50 & 0 \\
\hline Cefotaxime & 100 & 50 & 0 \\
\hline Cefaclor & 100 & 5 I & 0 \\
\hline Ciprofloxacina & 29 & 36 & 4 \\
\hline Fosfomicina & 95 & 58 & 33 \\
\hline Trimet+Sulfam & 97 & 1 I & 2 \\
\hline Teicoplanina & 3 & 3 & 0 \\
\hline Vancomicina & 12 & 3 & 5 \\
\hline Tetraciclina & 80 & 27 & 82 \\
\hline
\end{tabular}

Tabella 3. Distribuzione delle percentuali di resistenza agli antibiotici tra i principali patogeni urinari isolati in ambiente comunitario e nosocomiale.

\begin{tabular}{|c|c|c|c|c|c|c|}
\hline & \multicolumn{2}{|c|}{ E. coli } & \multicolumn{2}{|c|}{ K. pneumoniae } & \multicolumn{2}{|c|}{ Proteus spp } \\
\hline Antibiotico & OSP & COM & OSP & COM & OSP & COM \\
\hline Ampicillina & 62.4 & 50.4 & 100 & 98.9 & 82.9 & 63.7 \\
\hline Amox+Clav. & 33.6 & 24.3 & 11.7 & 11.2 & 55 & 30.1 \\
\hline Amikacina & 1.2 & 0.7 & 1.2 & 1.3 & 0.6 & 1.8 \\
\hline Gentamicina & 15.3 & 9 & 5.8 & 3.3 & 25 & 30 \\
\hline Cefotaxime & 23.1 & 8.6 & 9.3 & 5.3 & 59.6 & 27.5 \\
\hline Ceftazidime & 22.8 & 8.9 & 10.6 & 6.1 & 55.8 & 22.2 \\
\hline Cefaclor & 61.6 & 51.8 & 18.8 & 11.3 & 70.2 & 40.7 \\
\hline Cefalotina & 61.3 & 43.3 & 24.3 & 19.1 & 72.4 & 47.9 \\
\hline Cefepime & 22.7 & 9.7 & 10.5 & 5.1 & 28.5 & 17.8 \\
\hline Ciprofloxacina & 40.8 & 27.8 & 10.3 & 4.9 & 62.3 & 41.5 \\
\hline Norfloxacina & 40 & 27.6 & 10.7 & 5.4 & 56.8 & 31.7 \\
\hline Fosfomicina & 14.9 & 9.1 & 15.8 & 37.6 & 36 & 48.4 \\
\hline Piperacillina & 48.3 & 0.36 & 93.2 & 76.8 & 65.4 & 41 \\
\hline Piper+tazob & 7.8 & 3.5 & 6.8 & 5.3 & 4.4 & 4.8 \\
\hline Trimet+sulfam & 31 & 27.7 & 13.1 & 11.8 & 63.3 & 57.4 \\
\hline Nitrofurantoina & 7.2 & 6.4 & 57.8 & $60.10 \%$ & 99.3 & 100 \\
\hline
\end{tabular}

Osp. ospedaliero, Com. comunitario

per piperacillina, di circa il $50 \%$ per ampicillina. per Klebsiella spp e Proteus spp la sensibilità è risultata compresa tra il 95-99\% per piperacillina-tazobactam, gentamicina, e rispettivamente del $93 \%$ e compresa tra il $68-52 \%$ per le cefalosporine di III generazione cefotaxime e ceftazidime e per i chinolonici ciprofloxacina e norfloxacina (Tabella 1).

Considerando i Gram-positivi (Tabella 2) esiste un ampio margine di sensibilità ai glicopeptidi e all'amoxicillina clavulanato per gli enterococchi, mentre per gli stafilococchi,oltre ai glicopeptidi cotrimossazolo e tetraciclina potrebbero avere uno spazio terapeutico dopo le valutazioni del laboratorio. Per gli streptococchi solo la fosfomicina presenta qualche limite di refrattarietà.

Un'analisi comparativa tra i ceppi isolati in ambiente ospedaliero e quelli comunitari (Tabella 3), dimostra, come atteso, una maggior quota di ceppi sensibili agli antibiotici in quest'ultimo sito. Le variazioni in termini di percentuali sono in funzione del patogeno analizzato e del principio attivo considerato e possono variare dalle poche unità sino ad un aumento della sensibilità di circa il 50\%.

\section{CONCLUSIONI.}

Dai risultati ottenuti si evince che gli enterobatteri, con netta prevalenza di E. coli, causano la maggior parte delle IVU e che in vitro risultano sensibili soprattutto a piperacillina-tazobactam. Appare in aumento la resistenza ai fluorochinolonici per E.coli e Proteus spp comunitari. Queste osservazioni appaionoin linea con quelli della letteratura (1-4) e suggeriscono un aggiornamento continuo dei dati al fine di permettere il costante monitoraggio sul territorio degli enteropatogeni urinari di più frequente riscontro e la sorveglianza dell'antibiotico-resistenza.

\section{BIBLIOGRAFIA}

1. Boyd LB, Atmar RL, Randall GL, et al. Increased fluoroquinolone resistance with time in Escherichia coli from $>17.000$ patients at a large county hospital as a function of culture site, age, sex, and location. BMC Infect Dis. 2008; 15: 8-4.

2. Miragliotta G, Di Pierro MN, Miragliotta L, Mosca A. Antimicrobial resistance among uropathogens responsible for community-acquired urinary tract infections in an Italian community. J Chemother. 2008; 20: 721-7.

3. Tlamçani Z, Ellaia K, Benomar A, Kabbaj H, Alaoui A, Seffar M. Resistance to fluoroquinolone among Klebsiella spp strains producing extended-spectrum betalactamases isolated from urines. Ann Biol Clin (Paris). 2009; 67: 553-6.

4. Wada K, Kariyama R, Mitsuhata R, Uehara S, Watanabe T, Monden K, Kumon H. Experimental and clinical studies on fluoroquinolone-insusceptible Escherichia coli isolated from patients with urinary tract infections from 1994 to 2007. Acta Med Okayama. 2009; 63: 263-72. 